\title{
Anesthetic considerations for robotic surgery
}

\author{
Jeong Rim Lee $e^{1,2}$ \\ ${ }^{1}$ Department of Anesthesiology and Pain Medicine, Yonsei University College of Medicine, ${ }^{2}$ Anesthesia and Pain Research Institute, \\ Seoul, Korea
}

Recently, demand for minimally invasive surgery has increased greatly. As a result, robot-assisted techniques have gained in popularity, because they overcome several of the shortcomings of conventional laparoscopic techniques. However, robotic surgery may require innovations with regard to patient positioning and the overall arrangement of operative equipment and personnel, which may go against the conservative nature of anesthesia care. Anesthesiologists should become familiar with these changes by learning the basic features of robotic surgical systems to offer better anesthetic care and promote patient safety. (Korean J Anesthesiol 2014; 66: 3-11)

Key Words: General anesthesia, Remote operation.

Over the past decade, robot-assisted surgery has become widespread in a variety of operations. Basically, robotic surgery offers the benefits of laparoscopic surgery, such as improved cosmesis, reduced postoperative pain, and wound complications, and faster recoveries, with shorter hospital stays. Robotic surgery also overcomes some of the shortcomings of conservative laparoscopic or endoscopic techniques, such as the assistantdependent unstable video camera platform, two-dimensional view, restricted ergonomics of the surgeon, and instruments with limited degrees of freedom and the absence of wrist gear. Robotic systems now present three-dimensional views with magnification and tools with seven degrees of freedom that are capable of duplicating hand movements with high accuracy [1].

Several robotic systems have attempted to enter the field of robotic surgery. Presently, the da Vinci system of Intuitive Surgical is the only commercially available robotic system and is pre- dominant in Korea. Thus, this article will focus on the da Vinci system.

According to Intuitive Surgical, Korea, robotic surgery was first started in Korea in 2005 with a single da Vinci system; 10 prostatectomies, 10 gastrectomies, and 5 cholecystectomies were performed that year. Currently, in total, 38 da Vinci systems have been installed, and 27,000 surgeries have been performed, including 6,731 in 2012. The most commonly conducted surgery is the prostatectomy, followed by thyroidectomy, low anterior resection, and gastrectomy. Each has now been performed in more than 1,000 cases. Nephrectomies and cholecystectomies have also gained in popularity; performance of gynecological and transoral robotic surgery (TORS) has also increased recently.

The setting for robotic surgery requires specific considerations that differ from conservative surgical techniques. The modern anesthesiologist should keep abreast of these changes,

Received: October 10, 2013. Accepted: October 29, 2013.

Corresponding author: Jeong Rim Lee, M.D., Department of Anesthesiology and Pain Medicine, Yonsei University College of Medicine, 250, Seongsan-ro, Sinchon-dong, Seodaemun-gu, Seoul 120-752, Korea. Tel: 82-2-2227-3840, Fax: 82-2-2227-7897, E-mail: manya@yuhs.ac (c) This is an open-access article distributed under the terms of the Creative Commons Attribution Non-Commercial License (http:// creativecommons.org/licenses/by-nc/3.0/), which permits unrestricted non-commercial use, distribution, and reproduction in any medium, provided the original work is properly cited. 
and consider the impact on the anesthetic plan and patient safety. These issues will be reviewed in this article.

\section{Introduction to the da Vinci System}

The da Vinci Surgical System has three components: a surgical cart, an optical threedimensional vision tower, and a console. The surgical cart has four arms that can be manipulated by the surgeon in a console through real-time computer-assisted control. The first two arms represent the right and left arms of the surgeon, so they hold the instruments. The third arm positions the endoscope. The optional fourth arm enables the surgeon to hold another instrument or perform additional tasks, such as holding countertraction and following running sutures, thus eliminating the need for a patient-side surgeon. The surgical cart is heavy and bulky. Because of the proximity of the cart to the patient, the patient must be guarded against inadvertent contact due to the motions of the robotic arms.

Instruments that are installed in the surgical cart have seven degrees of freedom: arm movement up and down in the vertical plane, side to side in the horizontal plane, forward extension to reach an object and retraction backwards, rotation around the central axis, as when supinating and pronating the hand, the wrist movement of extension and flexion, lateral movement to the ulnar and radial side, and opening and closing the instrument for grasping [2]. This "EndoWrist" technology exceeds the capacity of a surgeon's hand in open surgery. Additionally, more than $6 \mathrm{~Hz}$ of hand tremor can be filtered, and motion scaling also can be invoked, up to a ratio of $5: 1$.

In most cases, two surgeons perform the operation. Besides the surgeon at the console, the other skilled assistant at the tableside places the trocars and connects them with the robotic arms, changes the robotic instruments, and manipulates additional endoscopic instruments.

The console is where the surgeon sits to view the operational field and controls the robotic arms performing the surgery [3]. In the console, the surgeon attaches his/her hands to the manipulator, and the hand motions are translated into surgical instrument motion. In addition, there are three foot-pedals for disengaging robotic motion, alternating between the robotic arms, adjusting the camera, and controlling the energy of electric cauterization or ultrasonic instruments. The system allows the surgeon to be physically remote from the patient and to control the surgery from the console.

The optical tower contains the computer equipment needed to integrate the left and right optical channels to provide stereoscopic vision. It also runs the software to control the kinetics of the robotic arms.

In summary, the benefits of the robotic surgical system are as follows: first, the operating surgeon has a unique, three- dimensional stereoscopic view of the operative field with adjustable magnification and a stable and directly controlled camera platform. Second, the surgeon also regains three extra degrees of motion that are lost with conventional laparoscopy. Finally, the surgeon benefits from tremor-filtering and motion-scaling [4].

\section{Brief Introduction to Robotic Surgery}

\section{Prostatectomy}

Robot-assisted laparoscopic radical prostatectomy (RALRP) is the most commonly performed robotic surgery. In the USA, more than $80 \%$ of prostatectomies are now conducted by robotic surgery, and the rate of growth has declined due to the near saturation [1]. Typical blood loss is $150-250 \mathrm{ml}$, although more is not unusual. An experienced surgeon can perform an easy prostatectomy in $2.5 \mathrm{~h}$.

Several studies have shown that RALRP reduces hospital stay duration, blood loss, postoperative pain, and provided a more rapid return of urinary function and higher rate of potency recovery. Additionally, control of cancer by RALRP has been found to be comparable to open surgery $[1,5,6]$.

\section{Other genitourinary surgeries: nephrectomy, cystectomy}

Other urological robotic surgeries have seen increases in numbers. Regarding radical nephrectomy, robot-assisted surgery failed to show any advantage compared with the laparoscopic method. However, in partial nephrectomy, it is thought to have advantages over the laparoscopic method [7]. Robot-assisted partial nephrectomy (RAPN) was associated with a significant reduction in blood loss and surgical complications, and with a shorter duration of hospitalization [8]. Moreover, it has been used to reduce the risk of renal damage by shortening the "warm ischemic time" [7]. To reduce the warm ischemic time, early unclamping, no vascular clamping, selective renal parenchymal clamping, and selective arterial clamping have been attempted; these are difficult to perform in laparoscopic surgery [7]. Compared with RALRP, a wider surgical field is required, and clashes between the robotic arms can be problematic. Thus, RAPN requires three robotic arms, and the fourth arm is generally not used to minimize external collisions [9].

Robot-assisted radical cystectomy (RARC) is still in early development, but early studies have shown a reduction in complications with equivalent oncological results [10]. Like other robotic surgeries, RARC is expected to have the potential to reduce transfusion and to provide a more rapid return of bowel function. Although the comparison of major complications differs between studies, RARC is known to be at least not worse 
than the open procedure. Cost, quality of life after surgery, and long-term oncological outcomes need further investigation [11].

\section{Gynecological surgery}

While the introduction of robotic surgery in the field of gynecology was later than in other fields, there has also been a substantial increase in recent years [1]. It is difficult to perform complex tasks such as lymph node removal and intracorporeal knot tying within the female pelvic cavity [12]. Laparoscopic gynecological surgeries thus have a slow learning curve, with associated complications, and demand high surgical skills, especially when the surgical anatomical field is affected by advanced pathology [13]. A robotic surgical system may offer many advantages over laparoscopic surgery with regard to these difficulties.

Hysterectomy, myomectomy, tubal re-anastomosis, radical hysterectomy, lymph node dissection, and sacrocolpopexy have been performed using robotic surgical systems [13].

Some case studies and retrospective cohort reviews have shown that robot-assisted gynecological surgeries resulted in reduced blood loss and shorter hospital stays than laparoscopic or open surgeries. However, no randomized controlled study has been performed to date.

\section{Abdominal surgery}

Not only hollow viscus, but also solid abdominal organs, including the liver, pancreas, and adrenal glands are included within the indications for robotic surgery. Among the many types of abdominal surgery, fundoplication, rectal resection, and gastric bypass have been particularly preferred for robotic surgery due to the difficulties in orientation and dexterity, especially in small spaces when suturing is needed or the instruments are at unergonomic angles [1].

During robot-assisted gastrectomy, the surgical cart is placed at the head side of the patient and the anesthetic workstation and anesthesiologist are on the far left side of the patient. The table is tilted in $15^{\circ}$ reverse Trendelenburg. With an experienced laparoscopic surgeon, it is possible to achieve comparable clinical outcomes with robot-assisted gastrectomy to a skilled laparoscopic surgeon [14].

Robot-assisted colorectal dissection has been found to be feasible and can be performed safely. Several benefits of robotic surgery can facilitate certain steps in colorectal procedures, including splenic flexure takedown, dissection of the inferior mesenteric vessels, autonomic nerve preservation, rectal mobilization, ureter and gonadal vessel identification, dissection in the narrow pelvis, and suturing [4]. However, prolonged operation durations have been reported due to maneuvers that require repositioning of the robot and its arms, such as splenic flexure mobilization and high ligation of the inferior mesenteric artery and vein, which add significant time to the surgical procedure $[2,15]$. Similar clinical results between robotic and laparoscopic colorectal surgery with regard to operation time, perioperative $\mathrm{Hb}$ changes, conversion rates, and oncological radicality have been reported [16]. The duration of hospital stay showed mixed results, and none of the studies so far has clearly demonstrated enhanced recovery after surgery [4]. The reported overall complication rate was $11 \%$, with two cases being robot-related, and the conversion rate was $6 \%$ among 288 robotic colorectal procedures [4]. Although the concept of robotic colorectal surgery seems appealing based on these findings, firm evidence to support widespread implementation remains scarce.

Although pancreatic and hepatic malignancies have been resected via robotic surgery and have shown safe and feasible results when compared with open surgery, existing outcome data are unclear.

\section{Robotic cardiac surgery}

Various cardiac surgeries, including mitral valve surgery, coronary revascularization, arrhythmia operation, left ventricular lead implantation, congenital heart surgery, and aortic valve replacement, have been performed with robot-assisted techniques. The list continues to grow [3].

Contraindications for robotic cardiac surgery are essentially identical to those for one-lung ventilation: severe pulmonary hypertension, dense pleural adhesions, recent coronary infarction or unstable coronary disease, and severe atherosclerosis precluding peripheral and endovascular cannulation.

Aortic occlusion methods include transthoracic aortic crossclamp and endoaortic balloon occlusion. The latter method has the risk of aortic dissection, increased morbidity, and higher cost [17].

\section{Robotic thoracic surgery}

Thoracic surgical procedures include thymectomy, mediastinal mass extirpation, fundoplication, esophageal dissection, esophagectomy, and pulmonary lobectomy. Few series of isolated case reports have been published to date.

Because lung isolation is mandatory for thymectomy or mediastinal tumor resection, left-sided double lumen tubes are commonly used. These operations require training for port placement, use of proper instruments and correct robotic arms, and proper positioning; the learning curve is steep. However, robot-assisted techniques can provide an ideal surgical approach for thymectomy and the resection of other mediastinal tumors [18].

Changes in patient positioning are required during robot as- 
sisted minimally invasive thoraco-laparoscopic esophagectomy, because it consists of three different phases. First is the thoracic phase, during which the patient is positioned in the left lateral decubitus position and the robotic system sits on the dorsocranial side of the patient. At this stage, the esophagus is resected en bloc with the surrounding lymph nodes. Second is the abdominal phase, when the patient is turned to the supine position and the stomach is mobilized. Then, a left-sided vertical incision along the sternocleidomastoid muscle is made and the cervical phase of the esophagectomy is performed. Finally, the resected specimen is removed through the widened left para-umbilical trocar port, and the gastric conduit is anastomosed with the cervical esophagus [19]. Although the conventional open transthoracic method is the first choice, this technique has its own important limitations and significant morbidity. There are great expectations for robot-assisted surgery with regard to reduced blood loss and shorter intensive care unit stays, although at this stage evidence of its superiority is lacking [19].

The performance of robot-assisted pulmonary resection is limited to a few centers. The advantages are not yet clearly defined. In one center, many cases of robotic pulmonary resection were converted to open thoracotomy due to prolongation of surgery and difficulty in the dissection of tenacious calcified lymph nodes [18].

Regarding cost, although robotic thoracic surgery is more expensive than endoscopic surgery, this is expected to be compensated for by decreased hospital stay durations [20]. Increased surgical times, requirements for increased numbers of skilled personnel, costs, and outcomes should be investigated and addressed [21].

\section{Transoral robotic surgery}

Currently, the number of TORSs continues to increase [22]. Traditional ENT operations require wider surgical exposure than the actual surgical field. However, the robotic surgical system, with a three-dimensional surgical field, can provide adequate depth using various endoscopes, cameras, and dual eyepieces. Thus, disfiguring mandibulotomies or tracheostomies can be avoided with TORS. Additionally, the risks of chemoradiation therapy may be reduced or avoided, and recovery of postoperative quality of life, such as speaking and eating, is known to better and more rapid [22].

Possible problems related to the insertion of the robotic arm into the intraoral space include facial skin lacerations, tooth injuries, mucosal lacerations, mandible fractures, cervical spine fractures, and ocular injuries. However, according to a study using cadavers, even under intentionally reckless conditions, the forces generated by the da Vinci Surgical System and the application of these forces to the cadaver was not found to cause severe injury. Attempts to cause tooth injury resulted in either instrument failure or tripping alarms in the robot. Furthermore, attempts to lift the cadaver's head resulted in tripping alarms in the robot and it going into a safe mode, precluding further movement of the robotic arms against resistance [23].

Radical tonsillectomy, tongue base resection, supraglottic laryngectomy, and phonomicrosurgery have been performed using the da Vinci system. Current indications for TORS are benign lesions of the oral cavity, larynx, and pharynx, and all T1 and T2 malignancies. Exclusion criteria are known to be pediatric diseases, lesions invading the mandible, and dental procedures.

Three arms are needed: two laterally placed to hold instruments, and a central arm to hold the endoscopic camera. The surgeon places a mouth gag or retractor, and the three sterilely draped robotic arms are placed into their surgical positions. After completion of the surgery, hemostasis is achieved with confirmation of the bloodless surgical field by a Valsalva maneuver. After final assessment of the airway, PVC tube exchange is performed when significant laryngopharyngeal edema or airway compromise is suspected [22].

Surgical time depends on the experience of surgeon. The learning curve is known to be steep, and operation experience significantly shortens the preoperative set-up time.

\section{Robotic surgery in pediatric patients}

Several robot-assisted surgeries have been performed safely in pediatric patients, including simple procedures, such as pyeloplasty, PDA closure, and nephrectomy, and more complex procedures, such as Bochdalek hernia repair, Kasai portoenterostomy, and choledochal cyst excision [24,25]. Because the working space is limited and the abdominal wall is thinner than in adults, proper positioning of ports is important and inadvertent visceral injury during port introduction and instrument manipulation should always be kept in mind [25]. With respect to pyeloplasty, hospital stay duration and postoperative pain were reduced compared with open surgery, but comparable to laparoscopic surgery [25]. The relatively limited selection of instruments and the size of the robotic device port sites, which should be separated by a minimal distance of $46 \mathrm{~cm}$, are emphasized as limitations for use in children [25].

\section{Anesthetic Considerations for Robotic Surgery}

\section{General aspects}

In robot-assisted operations, spatial restrictions due to the bulky equipment are a universal issue. After the robot has been positioned and engaged, the anesthesiologist is unable to read- 
ily access the patient. Thus, any lines, monitors, and patientprotective devices must be placed beforehand and should be secured to ensure no kinking or displacement [26]. It is impossible to allow changes in patient position or any kind of access to the patient if the robot is not detached first. Because this time delay in patient management may result in critical complications, especially in unhealthy patients or pediatric cases [24], early detection of any problems by the anesthesiologist and training of the surgical team for fast detachment of the robotic system in emergency situations is needed. Additionally, no type of movement is allowed during an operation. Movement of the patient while robotic instruments are docked could lead to tearing or puncturing of internal organs and vasculature, with potentially devastating consequences [20].

According to the type of operation, robotic surgery may require surgical positioning that is relatively extreme and steeper than in other conventional or laparoscopic surgery. These extreme positions increase the risk of patients sliding off the OR table, making the use of restraints inevitable. Some of these extreme positions may even cause physiological changes. Additionally, bulky robotic arms accompanied by extreme positioning and prolonged operation durations place patients at risk of positioning injuries [27]. The anesthesiologist should give attention to the robotic arms and the patient position to prevent pressure or crush injuries. In one center, positioning injuries were documented in $6.6 \%$ of 334 robot-assisted adult urological procedures [27]. Longer operation durations and worse patient conditions were found to be significant risk factors [27].

Some procedures, such as upper abdominal, thoracic, or head and neck surgeries, require the patient's airway to be situated away from the anesthesiologist and anesthesia workstation. During these procedures, access to the airway is almost impossible [2].

Robotic surgeries regarding intrathoracic or intra-abdominal pathologies require the use of $\mathrm{CO}_{2}$ pneumoperitoneum or capnothorax. Many complications related to these conditions have been noted during laparoscopic surgery, such as subcutaneous emphysema, pneumothorax, pneumomediastium, and, in the worst case, gas embolism.

\section{Specific Anesthetic Considerations for Each Surgery}

\section{Prostatectomy}

Two main concepts associated with robotic prostatectomy are the steep Trendelenburg position and $\mathrm{CO}_{2}$ pneumoperitoneum. Several issues regarding them have been noticed: RALRP requires a much steeper Trendelenburg position and a higher pressure of $\mathrm{CO}_{2}$ pneumoperitoneum. Retroperitoneal dissection also increases the absorption of $\mathrm{CO}_{2}$ [28].

Interpreting the effect of the steep Trendelenburg position and that of $\mathrm{CO}_{2}$ pneumoperitoneum separately is impossible: the combination of the factors affects the patient additionally or synergistically. The Trendelenburg position combined with pneumoperitoneum pushes the abdominal contents cephalad; hence, FRC and lung compliance are reduced. However, the lung has a remarkable, though incompletely understood, capacity to withstand the effects of $\mathrm{CO}_{2}$ pneumoperitoneum and the steep Trendelenburg position during general anesthesia. While individual responses vary and should be monitored, effects on dead-space ventilation and venous admixture are typically small and should not be an obstacle to providing optimal surgical exposure during robot-assisted prostatectomy or hysterectomy [29]. Also, most of the lung is below the left atrium and thus in the pulmonary zone 3 or 4 condition. Patients are prone to ventilation-perfusion mismatch, atelectasis, and pulmonary interstitial edema [26]. To overcome the negative influence of the steep Trendelenburg position to the lungs, a tidal volume of 6-8 $\mathrm{ml} / \mathrm{kg}$ and a positive end-expiratory pressure of $4-7 \mathrm{cmH}_{2} \mathrm{O}$ are recommended for the prevention of atelectasis, and maximal airway pressure should be kept under $35 \mathrm{cmH}_{2} \mathrm{O}$ [12]. Pressurecontrolled ventilation was found to result in greater dynamic compliance and lower peak inspiratory airway pressure, compared with volume-controlled ventilation [30]. A prolonged inspiratory duration can be an alternative to producing better gaseous exchange conditions and respiratory mechanics [31]. I : E ratios of $2: 1$ or $1: 1$ provided better oxygenation and lower $\mathrm{PaCO}_{2}$ levels than the conventional $1: 2$ ratio by preserved $\mathrm{Vd} / \mathrm{Vt}$ in the steep Trendelenburg position. Hong et al. [32] recommended thoracic epidural anesthesia combined with general anesthesia. Epidural anesthesia was found to lower peak inspiratory airway pressure, enhance dynamic compliance, allow better oxygenation and lower concentrations of lactate through more profound muscle block, having a systemic stabilizing effect on the neuromuscular junction by local anesthetics, and attenuating bronchial hyperactivity [32].

The combination of the steep Trendelenburg position with $\mathrm{CO}_{2}$ pneumoperitoneum can reduce the length of the trachea. The distance from the vocal cord to the carina was reduced by about $1 \mathrm{~cm}$ compared to pre-positioning [33].

Regarding the cardiovascular system, central venous pressure, pulmonary artery pressure, and pulmonary capillary wedge pressure are increased according to the degree of head-down tilt [34], and, thus, the heart rate is decreased. Especially during the initiation of $\mathrm{CO}_{2}$ insufflation, severe bradycardia and asystole have been noted [6]. CO and CI, measured with Flotrac, showed slight decreases during the head-down position with pneumoperitoneum [28]. This is due to increased systemic vascular resistance (SVR) and afterload, and, therefore, reduced stroke 
volume. Falabella et al. [35] used transesophageal echocardiography, and showed that this position increased mean arterial pressure and SVR. While the Trendelenburg position itself may increase cardiac output, due to an increase in venous return, aortic compression by pneumoperitoneum increases SVR and thus the final stroke volume and cardiac output may decrease, rather than increase [35]. These combined results may lead to an increase in myocardial oxygen demand, and therefore caution is needed in patients with a reduced cardiac reservoir or impaired baroreflex [36].

The Trendelenburg position increases intracranial pressure $[37,38]$ and intraocular pressure (IOP) [39]. IOP was found to be increased by an average of $13 \mathrm{mmHg}$ after being positioned in the $25^{\circ}$ Trendelenburg position with $15 \mathrm{mmHg}$ of $\mathrm{CO}_{2}$ pneumoperitoneum, compared with the preinduction value, with surgical duration and $\mathrm{ETCO}_{2}$ being significant predictors [39]. Cerebral blood flow-carbon dioxide reactivity does not change under the steep Trendelenburg position with pneumoperitoneum during sevoflurane anesthesia [40], and cerebral oxygen saturation, measured with near-infrared spectroscopy, was increased slightly during surgery, mainly related with $\mathrm{PaCO}_{2}$ [41]. Jugular venous oxygen saturation does not seem to reflect brain oxygenation better than $\mathrm{rSO}_{2}$ in this condition [42]. This condition was also not found to compromise cerebral perfusion $[43,44]$. Most importantly, all of these non-physiological conditions were still within normal limits, and therefore well tolerated by most patients [34,44].

A unique consideration during $\mathrm{CO}_{2}$ insufflation is a variety of gas-related complications. Regarding gas embolisms, the incidence of venous gas embolism is less in RALRP than RRP ( $38 \%$ vs. $80 \%$ ) [45]. The period of transection of the deep dorsal venous complex, rather than the initiation of insufflation, is more likely to be the risky period for gas embolism. Subcutaneous emphysema is also a common complication [46]. Although subcutaneous emphysema itself is not a dangerous complication and is quickly resolved after cessation of insufflation, mechanical ventilation should be continued until hypercarbia is corrected to prevent any excessive increase in the work of breathing [46]. The possibility of pneumothorax or pneumomediastium should always be considered. Additionally, because $\mathrm{CO}_{2}$ readily permeates into the blood stream, the resulting hypercarbia may cause sympathetic stimulation, leading to increased heart rate and blood pressure [12]. In a case of severe gas embolism, with cardiovascular collapse, resuscitation is restricted due to the docking of the robot. The surgical team should practice the emergency drill for de-docking the robotic system if necessary to prevent a delay in resuscitation [12].

Clinical swelling of the face and upper airway with venous stasis in the head and neck is common [26]. Mildly restrictive fluid management is required for less facial edema and de- creased excessive urine output that could obscure the operation field $[6,47]$. One author suggested that no more than $800 \mathrm{ml}$ of fluid be given until the surgeon completes the vesico-urethral anastomosis, which should then be followed by an infusion of an additional 700-1,200 $\mathrm{ml}$ of fluid [46]. Lingual and buccal nerve neuropathies are possible. Before anesthetic emergence and extubation, the significance of any upper airway edema should be evaluated [47]. The steep Trendelenburg position is also prone to devastating ischemic optic neuropathy and corneal abrasions [46]. To prevent corneal abrasion, the incidence of which is $3 \%$, taping the eyes closed using a transparent occlusive dressing is required [6].

Careful positioning is essential. For RALRP, the patient is in a lithotomy and steep Trendelenburg position, of usually $30^{\circ}$ or more. The patient's arms will be tucked at the sides and the drapes will keep the patient far away from the reach of the anesthesiologist [26]. Such patients are prone to sliding off, but shoulder braces can cause brachial plexus injuries [47]. Therefore, the technique of strapping the patient to the operating table with chest banding is advocated. However, this method can aggravate the decrease in lung compliance [6]. This position can also injure the upper extremities by causing peripheral nerve injuries [46].

\section{Other urological surgeries}

For RARC, anesthetic considerations are similar to those for robotic prostatectomy, except that there is less volume restriction. RAPN is performed in a $45^{\circ}$ lateral decubitus position with flank elevation.

\section{Gynecological surgery}

Because of the similar positioning and pneumoperitoneum, most concerns regarding anesthesia are the same as for a prostatectomy. A less-steep Trendelenburg position, compared with urological surgery, is permitted [12].

\section{Transoral robotic surgery}

Basically, anesthesia considerations for TORS are similar to transoral cases, such as tonsillectomies and laser excision of laryngeal lesions. However, several points must be considered. The patient is turned $180^{\circ}$ away from the anesthesia workstation, and a fairly large device is placed in the vicinity of the patient's head. Additionally, the patient is placed in suspension laryngoscopy. The surgeon sits out of the view of the anesthesia team. During the operation, the eyes should be secured with patient safety goggles and the teeth should be protected with a molded dental guard. The endotracheal tube is sutured to the patient's 
face by the surgeon. A wire-reinforced tube is commonly used and, sometimes, a laser tube may be used [22].

Because advanced and extensive lesions with deep structure invasion are not candidates for TORS, difficult exposure in laryngoscopies is of less concern and invasive lines are not considered. However, an anesthesiologist has to know that some operations have the risk of rupture of the lingual artery, and tonsillectomy requires dissection to the plane of the carotid artery [22].

If anesthesia is induced with the bed turned $180^{\circ}$ at the outset, other preparations, including the robotic side cart and scrub table, can be easier, although induction may be more complicated. Avoiding bed turning not only saves time but also reduces the risk of disconnecting monitors and IV lines. Additionally, the patient's head should be positioned at the foot of the bed so that the pedestal of the bed is in the proper position when bringing the robotic cart into place [22].

In some centers, when the dissection is adjacent to the vallecular or epiglottis, in a prolonged case in which the tongue base is suspected, and in a supraglottic partial laryngectomy, the patient is intubated during the postoperative period for 1.5 days for airway evaluation and management. Indications for tracheostomy are resections that involve both the tongue base and a portion of the epiglottis, any patient in whom the management of a postoperative emergency situation is expected to be difficult, and other medical indications. It is recommended that all patients be given dexamethasone $10 \mathrm{mg}$ daily from the outset of surgery, to be continued until discharge [22].

Reported complications are rare, but include tracheostomy and bleeding. The incidence is comparable with other alternative therapies of non-robotic transoral surgery, open surgery, and chemotherapy with radiation therapy.

Neck dissection can be performed as a separate procedure after TORS, allowing for a shorter operation time, decreased tissue manipulation, and minimized laryngopharyngeal swelling. Some centers advocate neck dissection at the time of TORS.

\section{Robotic cardiac surgery}

For most robotic cardiac surgeries, the patient is in the supine position. The arm on the side of the chest port placement is allowed to hang over the edge of the table. Pads are placed below the chest to elevate the hemithorax by $25-30^{\circ}$ to allow port placement in a triangular arrangement. External defibrillation is essential, and the patches and ECG electrodes should be away from the site of port placement [48].

Single-lung ventilation is required for most robotic cardiac surgeries and sometimes $\mathrm{CO}_{2}$ insufflation is added to provide a better surgical field. These situations cause hypercapnia and impede venous return, thus reducing cardiac output and leading to possible acute cardiovascular collapse [17]. To avoid such situations, $\mathrm{CO}_{2}$ insufflation should be started $30-60 \mathrm{~s}$ after pleural opening to ambient air at a slow rate $(1 \mathrm{~L} / \mathrm{min})$ [20]. Changes in the electrical axis after capnothorax may induce electrocardiography changes, which should be considered in the differential diagnosis.

Cardiopulmonary bypass is achieved with femoral artery and venous cannulation, and a $17-\mathrm{F}$ right internal jugular venous cannulation is needed for venous drainage. In some cases, the pulmonary artery vent and coronary sinus cardioplegia catheter are secured by the anesthesiologist. A transesophageal echocardiogram is helpful for guiding venous cannulation, positioning the PAC for venting the pulmonary artery, placing a coronary sinus catheter, and assessing atherosclerosis in the descending aorta. For measuring blood pressure, transthoracic aortic or axillary artery cannulation may be appropriate for patients with peripheral vascular disease. If the radial artery must be cannulated, the right radial artery is preferred to detect migration of the endovascular balloon cannula causing obstruction of the innominate artery [3]. After cardiopulmonary bypass, de-airing is difficult due to lack of direct access to the heart for the surgeon and the slight lateral tilted position. Use of $\mathrm{CO}_{2}$ tends to displace air from the exposed areas of the heart [48].

Patient immobility must be absolutely guaranteed by pharmacological paralysis. Otherwise, tearing or puncturing of intrathoracic organs and vasculature can have devastating consequences [20].

\section{Robotic thoracic surgery}

For thymectomy, the patient is placed in an incomplete sideup position, at a $30^{\circ}$ right or left lateral decubitus position. The arm of the elevated side is positioned at the patient's side, as far back as possible, so the surgeon can gain enough space for the robotic arms [21]. This increases the concern for brachial plexus injury and special attention must be given to the elevated arm or head to prevent crushing injuries by the robotic arms. $\mathrm{CO}_{2}$ insufflation to a pressure of $10-15 \mathrm{mmHg}$ can be applied to keep the lung away from the surgical field, but it can obstruct venous return and cause profound hypotension. The key issue for anesthesia during one-lung ventilation and capnothorax is maintaining stable hemodynamics and oxygenation [48]. Careful selection of appropriate patients with proper preoperative testing is important.

For esophagectomy, the operation consists of three stages. The first is performed with robot assistance, and the left lateral decubitus position with a $45^{\circ}$ tilt toward the prone position is preferred.

For thoracic surgery in the lateral decubitus position, patient position also impairs venous return. The thorax is the highest 
point for maximal robotic arm range and maneuverability, so the lower extremities are below the level of the heart [20].

There is a high risk of positioning neuropathy during robotic thoracic surgery. Inadequate padding or positioning and inadvertent robotic arm placement can cause external nerve compression. During positioning, the arms and shoulders should be well cared for by reducing conflict with robotic arms and decreasing the risk of brachial plexus injury [17]. As in other robotic surgeries, the robotic arm monitors and surgical personnel will occupy the area around the patient, so extensions for IV lines are necessary, and injection ports or stopcocks need to be in accessible locations. Long monitoring lines and anesthesia circuits are also mandatory [20]. During the surgical procedure, compression of cardiac or major vascular structures may also result in hemodynamic instability, with ECG changes [20].

There are several pitfalls to be considered regarding robotassisted surgery. First, the equipment is extremely bulky and thus considerable space is required. Second, the large size of the robot itself may result in collisions with its own arms, assistants, or patients. Third, it is difficult for the anesthesiologist to quickly access the patient during an operation. In addition, it is almost impossible to reposition the patient once the robot has been stationed.

Further research should be directed at addressing questions of long-term outcome, cost-effectiveness, effects on resident training, and whether this technology is best made available to all surgeons or to a limited number of surgeons with high surgical volume. Well-designed randomized trials regarding various types of surgeries with clinically meaningful long-term outcomes, such as quality of life and patient satisfaction, have to be conducted [13].

Nevertheless, the application of robot-assisted surgery will continue to increase and be extended to other fields. Anesthesiologists should stay up-to-date with this latest surgical trend and be ready to provide better anesthesia care for patients undergoing robot-assisted surgery.

\section{References}

1. Giri S, Sarkar DK. Current status of robotic surgery. Indian J Surg 2012; 74: 242-7.

2. Sullivan MJ, Frost EA, Lew MW. Anesthetic care of the patient for robotic surgery. Middle East J Anesthesiol 2008; 19: 967-82.

3. Chauhan S, Sukesan S. Anesthesia for robotic cardiac surgery: an amalgam of technology and skill. Ann Card Anaesth 2010; 13: 169-75.

4. Mirnezami AH, Mirnezami R, Venkatasubramaniam AK, Chandrakumaran K, Cecil TD, Moran BJ. Robotic colorectal surgery: hype or new hope? A systematic review of robotics in colorectal surgery. Colorectal Dis 2010; 12: 1084-93.

5. D'Alonzo RC, Gan TJ, Moul JW, Albala DM, Polascik TJ, Robertson CN, et al. A retrospective comparison of anesthetic management of robot-assisted laparoscopic radical prostatectomy versus radical retropubic prostatectomy. J Clin Anesth 2009; 21: 322-8.

6. Gainsburg DM, Wax D, Reich DL, Carlucci JR, Samadi DB. Intraoperative management of robotic-assisted versus open radical prostatectomy. JSLS 2010; 14: 1-5.

7. Png KS, Sundaram CP. Current status of robot-assisted laparoscopic partial nephrectomy. Indian J Surg Oncol 2012; 3: 91-5.

8. Vittori G. Open versus robotic-assisted partial nephrectomy: a multicenter comparison study of perioperative results and complications. World J Urol 2013 [Epub ahead of print].

9. Wang L, Lee BR. Robotic partial nephrectomy: current technique and outcomes. Int J Urol 2013; 20: 848-59.

10. Liss MA, Kader AK. Robotic-assisted laparoscopic radical cystectomy: history, techniques and outcomes. World J Urol 2013; 31: 489-97.

11. Yuh BE, Nazmy M, Ruel NH, Jankowski JT, Menchaca AR, Torrey RR, et al. Standardized analysis of frequency and severity of complications after robot-assisted radical cystectomy. Eur Urol 2012; 62: 806-13.

12. Gupta K, Mehta Y, Sarin Jolly A, Khanna S. Anaesthesia for robotic gynaecological surgery. Anaesth Intensive Care 2012; 40: 614-21.

13. Visco AG, Advincula AP. Robotic gynecologic surgery. Obstet Gynecol 2008; 112: 1369-84.

14. Song J, Kang WH, Oh SJ, Hyung WJ, Choi SH, Noh SH. Role of robotic gastrectomy using da Vinci system compared with laparoscopic gastrectomy: initial experience of 20 consecutive cases. Surg Endosc 2009; 23: 1204-11.

15. Wexner SD, Bergamaschi R, Lacy A, Udo J, Brölmann H, Kennedy RH. The current status of robotic pelvic surgery: results of a multinational interdisciplinary consensus conference. Surg Endosc 2009; 23: 438-43.

16. Baik SH, Ko YT, Kang CM, Lee WJ, Kim NK, Sohn SK. Robotic tumor-specific mesorectal excision of rectal cancer: short-term outcome of a pilot randomized trial. Surg Endosc 2008; 22: 1601-8.

17. Lehr EJ, Rodriguez E, Chitwood WR. Robotic cardiac surgery. Curr Opin Anaesthesiol 2011; 24: 77-85.

18. Cerfolio RJ, Bryant AS, Minnich DJ. Starting a robotic program in general thoracic surgery: why, how, and lessons learned. Ann Thorac Surg 2011; 91: 1729-36.

19. van der Sluis PC, Ruurda JP, van der Horst S, Verhage RJ, Besselink MG, Prins MJ, et al. Robot-assisted minimally invasive thoracolaparoscopic esophagectomy versus open transthoracic esophagectomy for resectable esophageal cancer, a randomized controlled trial (ROBOT trial). Trials 2012; 13: 230.

20. Steenwyk B, Lyerly R 3rd. Advancements in robotic-assisted thoracic surgery. Anesthesiol Clin 2012; 30: 699-708. 
21. Campos JH. An update on robotic thoracic surgery and anesthesia. Curr Opin Anaesthesiol 2010; 23: 1-6.

22. Chi JJ, Mandel JE, Weinstein GS, O'Malley BW Jr. Anesthetic considerations for transoral robotic surgery. Anesthesiol Clin 2010; 28 : 411-22.

23. Hockstein NG, O'Malley BW Jr, Weinstein GS. Assessment of intraoperative safety in transoral robotic surgery. Laryngoscope 2006; 116: 165-8.

24. Mariano ER, Furukawa L, Woo RK, Albanese CT, Brock-Utne JG. Anesthetic concerns for robot-assisted laparoscopy in an infant. Anesth Analg 2004; 99: 1665-7.

25. van Haasteren G, Levine S, Hayes W. Pediatric robotic surgery: early assessment. Pediatrics 2009; 124; 1642-9.

26. Baltayian S. A brief review: anesthesia for robotic prostatectomy. J Robotic Surg 2008; 2: 59-66.

27. Mills JT, Burris MB, Warburton DJ, Conaway MR, Schenkman NS, Krupski TL. Positioning injuries associated with robotic assisted urological surgery. J Urol 2013; 190: 580-4.

28. Darlong V, Kunhabdulla NP, Pandey R, Chandralekha, Punj J, Garg R, et al. Hemodynamic changes during robotic radical prostatectomy. Saudi J Anaesth 2012; 6: 213-8.

29. Schrijvers D, Mottrie A, Traen K, De Wolf AM, Vandermeersch E, Kalmar AF, et al. Pulmonary gas exchange is well preserved during robot assisted surgery in steep Trendelenburg position. Acta Anaesthesiol Belg 2009; 60: 229-33.

30. Choi EM, Na S, Choi SH, An J, Rha KH, Oh YJ. Comparison of volume-controlled and pressure-controlled ventilation in steep Trendelenburg position for robot-assisted laparoscopic radical prostatectomy. J Clin Anesth 2011; 23: 183-8.

31. Kim WH, Hahm TS, Kim JA, Sim WS, Choi DH, Lee EK, et al. Prolonged inspiratory time produces better gas exchange in patients undergoing laparoscopic surgery: A randomised trial. Acta Anaesthesiol Scand 2013; 57: 613-22.

32. Hong JY, Lee SJ, Rha KH, Roh GU, Kwon SY, Kil HK. Effects of thoracic epidural analgesia combined with general anesthesia on intraoperative ventilation/oxygenation and postoperative pulmonary complications in robot-assisted laparoscopic radical prostatectomy. J Endourol 2009; 23: 1843-9.

33. Chang CH, Lee HK, Nam SH. The displacement of the tracheal tube during robot-assisted radical prostatectomy. Eur J Anaesthesiol 2010; 27: 478-80.

34. Lestar M, Gunnarsson L, Lagerstrand L, Wiklund P, Odeberg-Wernerman S. Hemodynamic perturbations during robot-assisted laparoscopic radical prostatectomy in 45 degrees Trendelenburg position. Anesth Analg 2011; 113: 1069-75.

35. Falabella A, Moore-Jeffries E, Sullivan MJ, Nelson R, Lew M. Cardiac function during steep Trendelenburg position and CO2 pneumoperitoneum for robotic-assisted prostatectomy: a trans-oesophageal Doppler probe study. Int J Med Robot 2007; 3: 312-5.

36. Naylor JM, Chow CM, McLean AS, Heard RC, Avolio A. Cardiovascular responses to short-term head-down positioning in healthy young and older adults. Physiother Res Int 2005; 10: 32-47.

37. Josephs LG, Este-McDonald JR, Birkett DH, Hirsch EF. Diagnostic laparoscopy increases intracranial pressure. J Trauma 1994; 36: 815-8.

38. Irgau I, Koyfman Y, Tikellis JI. Elective intraoperative intracranial pressure monitoring during laparoscopic cholecystectomy. Arch Surg 1995; 130: 1011-3.

39. Awad H, Santilli S, Ohr M, Roth A, Yan W, Fernandez S, et al. The effects of steep trendelenburg positioning on intraocular pressure during robotic radical prostatectomy. Anesth Analg 2009; 109: 473-8.

40. Choi SH, Lee SJ, Rha KH, Shin SK, Oh YJ. The effect of pneumoperitoneum and Trendelenburg position on acute cerebral blood flowcarbon dioxide reactivity under sevoflurane anaesthesia. Anaesthesia 2008; 63: 1314-8.

41. Park EY, Koo BN, Min KT, Nam SH. The effect of pneumoperitoneum in the steep Trendelenburg position on cerebral oxygenation. Acta Anaesthesiol Scand 2009; 53: 895-9.

42. Choi SH, Kim SH, Lee SJ, Soh SR, Oh YJ. Cerebral oxygenation during laparoscopic surgery: jugular bulb versus regional cerebral oxygen saturation. Yonsei Med J 2013; 54: 225-30.

43. Kalmar AF, Dewaele F, Foubert L, Hendrickx JF, Heeremans EH, Struys MM, et al. Cerebral haemodynamic physiology during steep Trendelenburg position and CO2 pneumoperitoneum. Br J Anaesth 2012; 108: 478-84.

44. Kalmar AF, Foubert L, Hendrickx JF, Mottrie A, Absalom A, Mortier EP, et al. Influence of steep Trendelenburg position and CO2 pneumoperitoneum on cardiovascular, cerebrovascular, and respiratory homeostasis during robotic prostatectomy. Br J Anaesth 2010; 104: 433-9.

45. Hong JY, Kim JY, Choi YD, Rha KH, Yoon SJ, Kil HK. Incidence of venous gas embolism during robotic-assisted laparoscopic radical prostatectomy is lower than that during radical retropubic prostatectomy. Br J Anaesth 2010; 105: 777-81.

46. Gainsburg DM. Anesthetic concerns for robotic-assisted laparoscopic radical prostatectomy. Minerva Anestesiol 2012; 78: 596-604.

47. Phong SV, Koh LK. Anaesthesia for robotic-assisted radical prostatectomy: considerations for laparoscopy in the Trendelenburg position. Anaesth Intensive Care 2007; 35: 281-5.

48. Wang G, Gao C, Zhou Q, Chen T, Wang Y, Wang J, et al. Anesthesia management of totally endoscopic atrial septal defect repair with a robotic surgical system. J Clin Anesth 2011; 23: 621-5. 